\title{
A 2.4-GHz current-reuse OOK wake-up receiver for MICS applications
}

\author{
Hyun Kim and Hyunchol Shin ${ }^{\text {a) }}$ \\ High-Speed Integrated Circuits and Systems Lab., Kwangwoon University, \\ 20 Kwangwoon-Ro, Nowon-gu, Seoul 139-701, Korea \\ a)hshin@kw.ac.kr
}

\begin{abstract}
An ISM-band CMOS OOK receiver is presented as a possible wake-up receiver for implantable cardioverter telemtry system applications. It is based on the heterodyne architecture with uncertain IF. Current reuse technique is employed such that the LC VCO current is reused by the rest part of OOK receiver circuitry. Fabricated in $0.18 \mu \mathrm{m}$ CMOS, the receiver dissipates $1 \mathrm{~mW}$ from $1.8-\mathrm{V}$ supply. Test results show that the current-reuse OOK receiver is fully functional and meet the performance requirements. The proposed circuit structure can be effective for low-power OOK wake-up receiver design in implantable telemetry devices.
\end{abstract}

Keywords: MICS, on-off keying, current reuse, receiver

Classification: Integrated circuits

\section{References}

[1] P. D. Bradley: Proc. IEEE Biomedical Circuits and Systems Conf. (2006) 158.

[2] S. Kim and H. Shin: IEICE Trans. Electron. E95-C (2012) 968.

[3] K.-G. Park, C.-Y. Jeong, J.-W. Park, J.-W. Lee, J.-G. Jo and C. Yoo: IEEE Microw. Wireless Compon. Lett. 18 (2008) 413.

[4] D. Park, W. Lee, S. Jeon and S. Cho: Symp. VLSI Circuits Dig. Tech. Papers (2008) 88.

[5] K. W. Cheng, K. Natarajan and D. Allstot: IEEE J. Solid-State Circuits 45 (2010) 510.

[6] N. M. Pletcher, S. Gambini and J. Rabaey: IEEE J. Solid-State Circuits 44 (2009) 269.

[7] M. S. McCorquodale, J. D. O’Day, S. M. Pernia, G. A. Carichner, S. Kubba and R. B. Brown: IEEE J. Solid-State Circuits 42 (2007) 385.

[8] L. Fanori and P. Andreani: IEEE Int. Solid-State Circuits Conf. Dig. Tech. Papers (2013) 346.

[9] http://www.ti.com/product/cc2500

\section{Introduction}

A single-chip low-power RF transceiver in medical implant communication service (MICS) band is needed for implantable cardioverter defibrillators (ICD). It is used for exchanging data between the ICD and external 
controller, which is referred to as the implantable cardioverter telemetry system (ICTS). Typical ICTS transceiver comprises a MICS-band (402$405 \mathrm{MHz}$ ) main transceiver that sets the main telemetry link, and an additional ISM-band $(2.4 \mathrm{GHz})$ wake-up receiver that constantly monitors externally in-coming wake-up signal [1]. The wake-up signal is utilized for duty-cycling the main transceiver and thus lowering the average power consumption of the full system [1]. Since the wake-up receiver should be always operating not to miss any wake-up signal, its power dissipation sets the power dissipation floor of the total ICTS system. Hence, low power consumption is essential for the wake-up receiver design. On-off keying (OOK) modulation is preferred for the wake-up receiver due to its good energy efficiency and strong interferer tolerance. Therefore, along with a 400-MHz main transceiver, a low-power 2.4-GHz OOK receiver is essential block in ICTS.

Current reuse technique is effective for minimizing the total power dissipation. Many RF circuit blocks based on the current-reuse technique have been reported previously. It ranges from a single block circuit such as an LC VCO with a current-reusing p-FET and n-FET [2], to a composite block such as a VCO stacked on a frequency divider [3], a PLL stacked on a VCO [4], and further to a whole receiver chain such as the stacked LNA, mixer, and VCO [5]. All the works demonstrate significant reduction of current consumption while not compromising the circuit performances.

Since the key design challenge of the wake-up receiver is to decrease the power consumption for increasing the battery lifetime, the current reuse technique should be a good approach. However, such a current-reuse OOK receiver has never been reported before in the literature. This paper presents a 2.4-GHz ISM-band CMOS OOK receiver for an ICTS single-chip transceiver for ICD applications. It is based on the current reuse technique by stacking an LC VCO on all the rest part of the OOK receiver circuits.

\section{Circuit design and results}

Fig. 1 shows the OOK receiver architecture. It comprises LNA, mixer, IF amplifier, envelope detector, and LC VCO. As can be seen, the VCO resides over the rest circuits and shares the bias current. The receiver is based on the heterodyne architecture with uncertain intermediate frequency (IF) [6]. Incoming OOK-modulated RF signal is first amplified by the LNA for enhancing the sensitivity and subsequently down-converted to IF. The IF OOK signal is then demodulated to the baseband by the envelope detector.

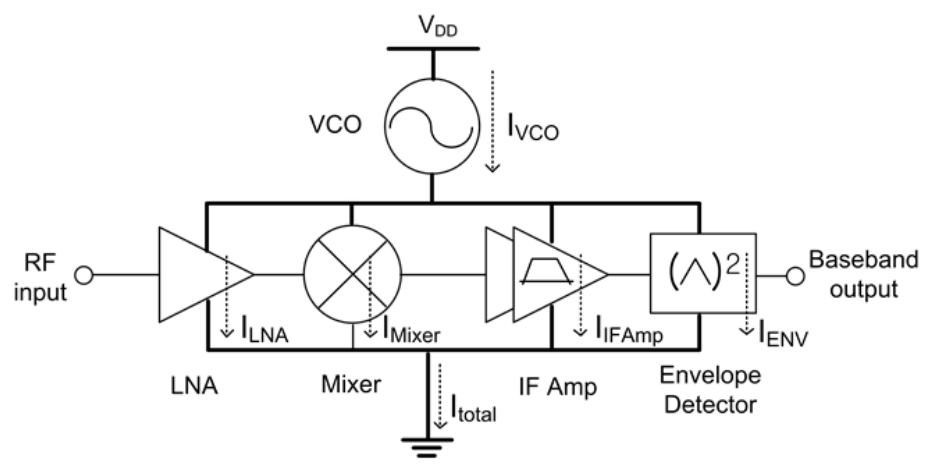

Fig. 1. Current-reuse OOK receiver. 
The RF frequency is the ISM-band frequency of $2.45 \mathrm{GHz}$. The IF frequency is typically set to $100 \mathrm{MHz}$. But it can also range between 50 and $200 \mathrm{MHz}$ without affecting the receiver operation. If we want to have a precise IF, an accurate LO frequency should be generated in the receiver. Such an accurate frequency generation will require a PLL with a crystal reference oscillator, which will inevitably worsen the system form factor and power dissipation of the total receiver. Thus, only moderately precise (or imprecise) LO generation from a stand-alone VCO (without a PLL and a crystal reference) is desired for ultra-low power wireless sensor network applications. In [6], a ring oscillator was adopted for the LO generation. However, it needed an external frequency calibration to be performed periodically, which could be a system overhead. In this work, an LC VCO is employed since it is known that the LC VCO can provide higher accuracy up to several hundreds of ppm over process, voltage, and temperature variation only with a one-time factory calibration and no further subsequent calibration [7].

Fig. 2 shows the circuit schematic of the total receiver. The supply voltage $\mathrm{V}_{\mathrm{DD}}$ is $1.8 \mathrm{~V}$, and the intermediate supply voltage $\mathrm{V}_{\text {mid }}$ is chosen as $1.2 \mathrm{~V}$. The intermediate capacitor $\mathrm{C}_{\text {mid }}$ regulates $\mathrm{V}_{\text {mid }}$ and minimizes the second-harmonic ripple noise at the node. $\mathrm{C}_{\text {mid }}$ is set to $40 \mathrm{pF}$ that comprises MIM and MOS capacitors. The current consumption of each block is $240 \mu \mathrm{A}$ for LNA, $80 \mu \mathrm{A}$ for mixer, $160 \mu \mathrm{A}$ for two-stage IF amplifier, $50 \mu \mathrm{A}$ for envelope detector, and $10 \mu \mathrm{A}$ for three-stage output buffer, all of which add up to $560 \mu \mathrm{A}$ that is reused by the VCO. The VCO operates with $0.6 \mathrm{~V}$ effective supply voltage. Even with such a relatively low supply voltage, the VCO generates sufficiently large output swing for the mixer. It is because of the class-D operation. Class-D operation of the cross-coupled FET structure is capable of providing the maximum swing up to 3.2 times the gate-source bias voltage [8]. In the simulation, the actual output swing of the VCO is found to be much lower $0.6 \mathrm{~V}_{\mathrm{p}-\mathrm{p}}$, which is however sufficient for the mixer switching. The VCO tank inductor is $4.4 \mathrm{nH}$ and the switched capacitor bank allows the frequency tuning between 2.2 and $2.6 \mathrm{GHz}$.

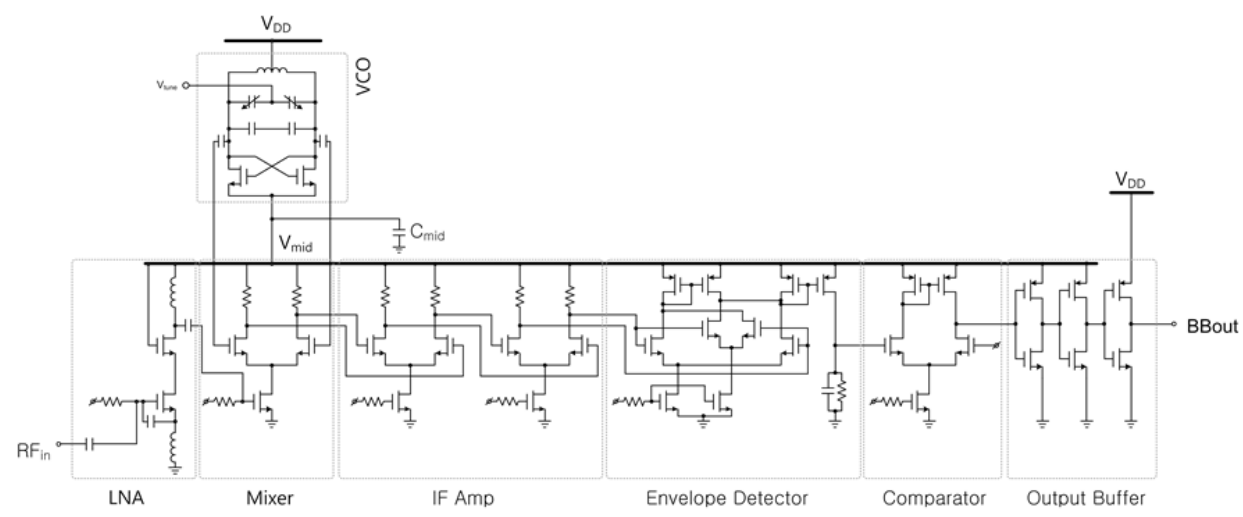

Fig. 2. Circuit schematic.

The cascode LNA employs an additional gate-source capacitance $80 \mathrm{fF}$ and a source inductance $1.2 \mathrm{nH}$ for minimum noise figure. The load inductance is set to $5.78 \mathrm{nH}$ for maximum gain. The gain and noise figure is $15 \mathrm{~dB}$ and $3.6 \mathrm{~dB}$, respectively, in simulation. A single-balanced type active switching mixer is chosen instead of the double-balanced type because it 
saves the current dissipation and the LO and RF leakage can be easily suppressed by the subsequent IF circuits. The two-stage IF amplifier employs resistive load for simple biasing. The envelope detector is a fullwave current rectifier with RC load. The conversion gain and bandwidth are set by the RC load. The envelope detector output signal is compared with a preset dc reference voltage and discrimated to a binary signal. It is then amplified by the two-stage inverter buffer and finally converted to the 1.8-V digital baseband output.

The OOK receiver is fabricated in $0.18 \mu \mathrm{m}$ RF CMOS process. Fig. 3(a) shows the chip photo. The silicon area is $1.2 \mathrm{~mm}^{2}$ including pads. It consumes $560 \mu \mathrm{A}$ from a $1.8-\mathrm{V}$ supply voltage. S11 measurement shows the input return loss better than $-15 \mathrm{~dB}$ over the 2.4-GH ISM band. For verifying the OOK receiver operation, a commercial ISM-band OOK transmitter [9] is used. The OOK modulated signal as shown in Fig. 3(b) is transmitted through the air and received by the fabricated receiver chip. The RF and IF frequencies are $2.45 \mathrm{GHz}$ and $100 \mathrm{MHz}$, respectively. The data rate is $100 \mathrm{kbps}$. Fig. 3(c) shows the final demodulated baseband output from the receiver chip. As can be seen, the transmitted and received data indicated by the arrow line show exact agreement each other. The receiver sensitivity is $-60 \mathrm{dBm}$.

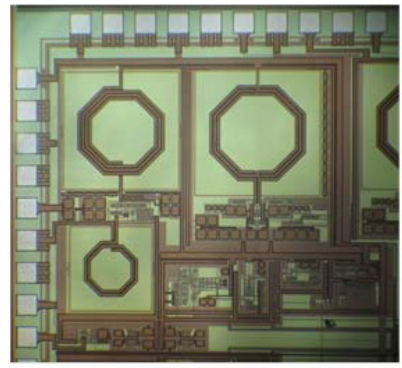

(a)

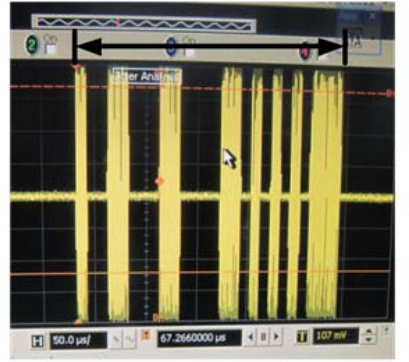

(b)

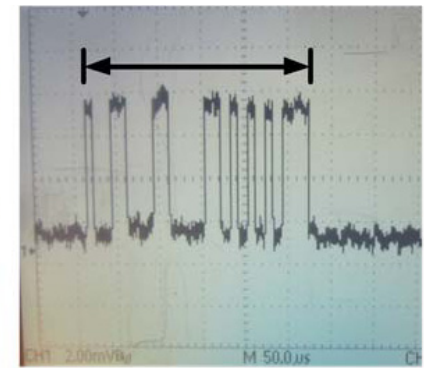

(c)

Fig. 3. (a) Chip micrograph. (b) Transmitted OOK signal from a test transmitter. (c) Received OOK signal from a fabricated receiver chip.

\section{Conclusion}

A 2.4-GHz ISM-band OOK receiver is presented for the ICTS wake-up receiver applications in ICD. The receiver is based on the heterodyne architecture with IF frequency ranging from 50 to $200 \mathrm{MHz}$. The total current of the whole OOK receiver circuitry is successfully reused by the VCO LO generation block, thus the current consumption of the receiver is reduced by half. Realized in $0.18 \mu \mathrm{m}$ CMOS, the receiver consumes $1 \mathrm{~mW}$ with 1.8-V supply. Test results show that the current-reuse OOK receiver is fully functional and meet the performance requirements.

\section{Acknowledgments}

This work has been supported by University ITRC program (NIPA-2013H0301-12-1013), and NRF Basic Science Research Program (2012038649), and Kwangwoon Research Grant 2013. 